\title{
Morphological and Morphometric Characterization of Indigenous Goat in Their Native Environment in West Omo and Bench-Sheko Zone, Southwestern Ethiopia
}

\author{
Shegaw Ambel ${ }^{*} \quad$ Elias Bayou $^{2}$ \\ 1. Animal Science, Mizan Agricultural Vocational, Educational and Training College, Ethiopia \\ 2. Department of Animal science, Mizan-Tepi University, Mizan Aman, Ethiopia \\ Crosponding Author, shegawambel@gmail.com
}

\begin{abstract}
This study was conducted on Morphological and Morph metric characterization of indigenous goat in West Omo and Bench-Sheko zone of in their native environment. About 660 goats were taken for body measurements of both sexes from 1PPI to 4PPI. Sex, districts and age class effect were considered in this study. The main frequently observed coat color patterns of goats were patchy and plain in Meanit Goldiya and Guraferda area, respectively. The main dominantly observed coat color type were white dominate with black for both sexes in Meanit Goldiya and brown for female and white for male goats were dominantly observed coat color in Guraferda area. Majority of goats had concave head profile in both districts. Body weight of goats increased as the age increased from 1pair of permanent incisor (PPI) to 4 PPI in the studied areas. Body weight and all the body measurements were significantly $(\mathrm{P}<0.01)$ affected by age group. Average body weight of males at age of 1PPI, 2PPI, 3PPI and 4PPI were $23.4 \pm 0.29,26.4 \pm 0.81,31.6 \pm 0.99$ and $34.0 \pm 0.55 \mathrm{~kg}$, respectively. The corresponding values for female goats were $20.2 \pm 0.12,23.6 \pm 0.44,26.2 \pm 0.18$ and $30.0 \pm 0.16 \mathrm{~kg}$, respectively. Therefore, the present study suggests that indigenous goat population might possess useful genetic potential for improving productivity under extensive feeding-based production system. Similarly, morphological and phonotypical variations have been observed between the indigenous goat populations; hence a profoundly molecular evaluation is needed to show the level of genetic differentiation and relation between them.
\end{abstract}

Keywords: body weight: Age, Morphological, characterization

DOI: $10.7176 / \mathrm{JBAH} / 12-3-04$

Publication date: February $28^{\text {th }} 2022$

\section{Introduction}

Ethiopia has an immense number of goats which are playing a great role in the economies of the country and the farmers at large. In traditional production systems, goats are not bred for a specific purpose rather they are kept for multipurpose functions. They provide multiple roles for their owners such as source of income, food (blood, meat and milk), fiber and skin, manure, insurance against crop failure and socio-cultural value (Legesse et al., 2008; Assen and Aklilu, 2012). The goat populations in Ethiopia are phenotypically classified into 12 distinct major breeds and categorized in to four families (Gizaw et al., 2010). However, farm animal breed characterization and identification work has been only identified eight goat breeds so far (EBI, 2016) with an estimated 32.7 million goats (CSA, 2018). According to this author, eight genetic entities include; Keffa, Abergalle, Afar, Highland goat, Eastern and south Easter goat, Gumez, Arsi-bale and woyto-Guji goat. Even though the enormous goat population and the roles of goats at farmers and national level, there is unsatisfactory efforts made to sustain gen-etic improvement programs, identification and structure of the goat populations. Amon-g them, one possible contributing factor for minimal benefit could be the absence of a clear breeding strategy to improve livestock production and productivity in Ethiopia livestock master plan (LMP, 2015).

Characterizations of farm animals are the primary footstep towards the utilization of the existing animal genetic resources, through either performance evaluation, phenotypic characterization and DNA or molecular characterization (FAO, 2010). In the same way goat, breed identification, documentation of their common uses and description of the management systems in which they are maintained are the first information to be assessed (FAO, 2011). These techniques provide essential indication for the distinction between and within domestic animal populations, which could be utilized for selection purposes (Okpeku et al., 2011). Lack of information on characterization of genetic resource may lead to undercutilization of that resource, its replacement and dilution through cross breeding despite their local adaptation to prevailing environmental constraints (Manzi et al., 2011). Detailed information provided by characterization need to be available to design a breeding and management programs. In general, breed characterization process is not exhaustive in Ethiopia. As a result, different research works (particularly phenotypic and genetic characterizations research activities) had been executed in different parts of the country by different organizations and individuals (Tesfaye, 2009; Tesfaye, 2010; Grum, 2010; Halima et al., 2012; Halima et al., 2012b). Despite the researches done, information on phenotypic characteristics of different indigenous goat population is still insufficient especially little was known regarding the phenotypic 
variability of goat gene-tic resource in this area.

Live weight measurement is a fundamental tool to known animal performance for provides reliable and informative information for selection, feeding requirements, health management, and decision on selling price (Thiruvenkanden, 2005). Therefore, under field condition, linear body measurements of various body conformations are an important valuable instrument to estimate indirect way of live weight(Tesfaye,2008), and are helpful in developing suitable selection criteria and judging quantitative characteristics of meat. In general, the information obtained in this study will be useful for designing appropriate community-based breeding program and sustainable conservation of goat genetic resource for indigenous goat improvement and wisely utilization of this resource. The objective of this study was to characterize morphologically and Morph metrically Meanit Goldiya and Guraferda goat in their home tract and production system.

\section{Material and Method}

\subsection{Site Selection of the study area}

The researcher was made rapid survey prior to the actual survey work to locate the distribution of goat in each district. The three kebeles from each district was selected purposively, which was based on the presence of indigenous got population, potential of goat production and location of the study area. For body linear measurements and qualities characteristics, a total 660 (600 adult female and 60 adult male) goats, 110 from each kebeles were selected within the selected sites.

Morph metric data collection

Based on breed morphological characteristics descriptor list of FAO (2012) for morphological characterization of goats, both qualitative and quantitative data were collected from 660 heads of adult goats. Data were recorded on the prepared format adopted from the standard description list developed by FAO (2012). Each experimental animal was identified by sex, districts and estimated age group. Dentition record was included, as this was the only reliable means to estimate the approximate age of an animal. Ages of the animals were estimated from dentition class following the procedure described by Wilson and Durkin (1984).

Adult goats were classified into four age groups; 1 PPI, 2 PPI, 3 PPI and 4 and above PPI following the description of African goat (Wilson and Durkin, 1984). Qualitative traits recorded during phenotypic characterization of goat breeds were sex, estimated age (dentition), coat color pattern and type, horn shape, horn and ear orientation, facial (head) and back profile as well as presence or absence of wattles, beard, and ruff. Whereas quantitative traits include the measurements of body length, chest girth, chest width, head length, rump length, pelvic width, horn length, ear length and scrotum circumference (for males only) were measured using tailors measuring tape while height at withers was measured using $2 \mathrm{~m}$ ruler. Body weight was measured using suspending balance having $50 \mathrm{~kg}$ capacity with $0.2 \mathrm{~kg}$ precision in the morning to avoid the effect of feeding and watering on the animal's size (FAO, 2012). Pregnant animals were excluded because pregnancy has effect on some morph metric parameters especially those of the thoracic and rump regions (Yakubu et al., 2011).

\subsection{Data Analysis}

\subsubsection{Morphological and body measurement data}

Observations on morphological characters were analyzed for male and female goat using frequency procedure of Statistical Analysis System (SAS Institute Inc., 2009).

Quantitative characters (body weight and linear body measurements) were analyzed using the Generalized Linear Model (GLM) procedures of the Statistical Analysis System (SAS Institute Inc., 2009).

For adult goat, sex and age group were fitted as fixed independent variables while body weight and linear body measurements except scrotum circumference was fitted as dependent variables. Scrotum circumference was analyzed by fitting age group as fixed factor. Least square means with their corresponding standard errors was calculated for each body trait over sex, age and age by sex interaction and means were separated using adjusted Tukey Kramer test.

Model for mean analysis in females and males except scrotal circumference were:

$Y i j k=\mu+D i+A j+S K+(A x S) j k+e i j k$

Where: Yijk = Observed body weight or linear measurements

$\mu=$ Overall mean

$\mathrm{Di}=$ the fixed effect of ith district (Guraferda and Meanit Goldiya)

$\mathrm{Aj}=$ the fixed effect of $\mathrm{jth}$ age classes (1PPI, 2PPI, 3PPI, 4 and above PPI)

$\mathrm{SK}=$ the fixed effects of kth sex (male, female)

(AS) ${ }_{j k}=$ the effect of interaction of $j$ of age group with $k$ of sex

eijk = random residual error

Model for mean analysis in males for scrotal circumference was:

$Y i j=\mu+D i+A j+e i j$

Where: $Y i j=$ Observed scrotum circumference 
$\mu=$ Overall mean

$\mathrm{Di}=$ the fixed effect of ith district (Guraferda and Meanit Goldiya)

$\mathrm{Aj}=$ the fixed effect of $\mathrm{jth}$ age classes (1PPI, 2PPI, 3PPI, 4 and above PPI)

eij= random residual error

Parameters were considered for goat live body weight and linear body measurements are: Pelvic Width (PW), Body Length (BL), Chest Girth (CG), Height at Wither (HW), Rump Length (RL), Ear Length (EL), Head length (HOL), Horn length (HL) and Scrotal Circumference (SC) for male, whereas Scrotum Circumference (SC) was excluded for the analysis means of parameters measured on female goats.

\section{Result}

\subsection{Morphological Characteristics}

The proportion of qualitative characters of goats in the study area is summarized in Table 1. In study area, majority $(49.7 \%)$ of the goat in Guraferda area were plain coat color pattern while $61.7 \%$ of goats in Meanit Goldiya were patchy and spotty coat col-or pattern. The majority goat population in Guraferda is mainly dominated by white (28.5) and brown (15.9\%) coat color type. The dominate coat color type in Meanit Goldiya was white dominate with black (29-.7\%), followed by black dominate with white $(21.9 \%)$. In studied sample population white spotted (light red) and patched with different color (black, grey or dark grey and brown), dark red, uniform black color goats were present with small and varied frequencies across populations. The majority (76.1- \%) of the goat had concave head profile across the studied areas. Majority of goat had straight back profile (76.7\%) followed by slopes up towards the rump (15.9\%). About $90.9 \%$ of goat had no wattle, $68.1 \%$ of the goats had no beard, $74.8 \%$ of had no ruff and $79.1 \%$ of the goat had no toggle. Goat with semi-pendulous ear type (55.7\%) and forward ear $(41.8 \%)$ were higher in proportion in the flock. Almost all $(99.9 \%)$ of goat had horn, of which $(62.9 \%)$ of goats were straight horn shape with back ward direction of $78.7 \%$, followed by curve-d $33.4 \%$, spiral $3.6 \%$.

\subsection{Morphometric Measurement}

The body weight and liner body measurements of goat by sex, age groups and district are presented in Table 2 . In the study area sex, district and age difference were clear for a various body measurement. The overall body weight goat, body length, chest girth, wither height, horn length, ear length, pelvic width, scrotum circumference, chest wid-th, head length and rump height in the study area was $27.3 \pm 0.18 \mathrm{~kg}, 60.1 \pm 0.16 \mathrm{~cm}, 70.7- \pm 0.16 \mathrm{~cm}$, $60.2 \pm 0.11 \mathrm{~cm}, \quad 9.2 \pm 0.08 \quad \mathrm{~cm}$ and $12.4 \pm 0.04 \quad \mathrm{~cm}, \quad 14.6 \pm 0.05 \quad \mathrm{~cm}, \quad 23.8 \pm 0.18$ $\mathrm{cm}, 14.4 \pm 0.05 \mathrm{~cm}, 11.06 \pm 0.05$ and $62.3 \pm 0.08 \mathrm{~cm}$, respectively.

Sex effect: Except ear length live body weight and all other body measurement were affected by sex $(P<0.05)$. The recorded body weight in this study for males and females was $29.91 \pm 0.67$ and $27.01 \pm 0.18 \mathrm{~kg}$, respectively. Male goats were consistently higher than females in all significantly affected variables except pelvic width (PW). Age effect: Body weight and all the body measurements were significantly $(P<0.01)$ affected by age group across the studied areas.BW, SC and CG were increased as the age increased from the youngest (1PPI) to the oldest (4 PPI) implied BW, SC and CG reached maximum at oldest age group. In other measurement (BL, WH, PW, RH, HOL.HL, EL and CW) intermediate age group (2-3 PPI) had larger values than the youngest age group (1PPI) but the values were the same with the old age group (4PPI).

District effect: variables (body weight, body length, wither at height, horn length, head length, ear length and chest width) were affected by district $(P<0.01)$, whereas, chest girth, rump height affected by district $(P<-0.05)$. Scrotum circumference of the goa-ts were not affected by district. Population in Guraferda had higher body weight, body length, wither at height, head length, ear length and chest width than population in Meanit Goldiya. The average body weight for Guraferda goat population was $28.19- \pm 0.28 \mathrm{~kg}$ which was higher than Meanit Goldiya goats $(26.46 \pm 0.2 \mathrm{~kg})$.

Sex by age group: The interaction of sex and age group was significant $(P<0.05)$ for $\mathrm{BW}, \mathrm{BL}, \mathrm{WH}, \mathrm{RH}$ and HOL but not significant $(P>0.05)$ for CG, PW, HL, EL and CW in the studied areas. In all age group males were heavier $(P<0.05)$ than females. CG was the same for male and female at youngest $(1 \mathrm{PPI})$ age group and males $C G$ was better at the intermediate (2-3PPI) and oldest (4PPI) age group. WH and RH was significantly $(P<0.05)$ larger for males than females in all age groups. Scrotum circumference was affected by the age of the male goat. SC at the age of 1 PPI was $21.3 \pm 0.19 \mathrm{~cm}$ which was significantly $(P<0.05)$ smaller than 2 PPI $(23.27- \pm 0.23 \mathrm{~cm}), 3$ PPI $(23.97- \pm 0.07 \mathrm{~cm})$ and 4 PPI $(25.2 \pm 0.11 \mathrm{~cm})$ age group. SC of the 4PPI age group were significantly $(P<0.05)$ larger than the 1PPI, 2PPI and 3PPI age group.

\section{Discussion}

Qualitative, quantitative and economic traits include under the phenotypic characterization of a particular breed (FAO, 2012). These characteristics are vital in breed identification, classification, decrepitation of management system and documentation of their common uses (FAO, 2011). The result obtained for plain coat color pattern of Guraferda goat is in a good agreement with the work of Tsigabu et al. (2015) in Nure goats in Gambela region, 
Ethiopia and Fantahun et al. (2013) in Bench Maji zone, southwestern. The major proportion obtain-ed for patchy coat color pattern of Meanit Goldiya goat is in comparable with the report of Netsanet (2014) in central high land goat. The value found for coat color type in Guraferda area was in comparable with the previous studies of Mahilet (2012) in Hararghe High land goat. Even though the percentages of some coat color type were small in a population, the present result showed that the studied goat populations have a wide range of coat color type which was in comparable with the previous finding of Halima et al. (2012) in Amhara region and Gurm (2010) around Dire Dawa. This indicate that farmers or goat keepers did not practices selection schemes for a particular trait and it create opportunities for develop breed improvements programs or upgrading breeds. The work of Mahilet (2012) in Haraghe High land, Netsanet (2014) in central high land goats and Tsigabu (2015) in Nure goats showed that majority of goat had concave head profile which is in accord-ance with the present finding. However, this result is disagreement with the previous study of Fantahun et al. (2013) in Bench Maji Zone Southwestern Ethiopia. Similar-ly, Tsigabu (2015) who stated that the back profile of goats had straight back in Nure Zone Gambella region which is in likely with the present study result. On the contrary, in Bench Maji zone majority goats had slopes up towards rump which is different from the current result (Fantahun et al., 2013). Majority of goats had no wattle, bear-d, ruff and toggle which is in agreement with the work of the earlier report of Fantahun et al. (2013) in Bench Maji area.

Table1.Percentages for each level of qualitative trait by goat population $($ male $=60$ and $\mathrm{Female}=600)$

\begin{tabular}{|c|c|c|c|c|c|c|c|c|}
\hline \multirow{4}{*}{$\begin{array}{l}\text { Character and } \\
\text { level }\end{array}$} & \multicolumn{6}{|c|}{ District } & \multirow{3}{*}{\multicolumn{2}{|c|}{ Test }} \\
\hline & \multicolumn{3}{|c|}{ Meanit Goldiya } & \multicolumn{3}{|c|}{ Guraferda } & & \\
\hline & Male & Female & Total & Male & Female & Total & & \\
\hline & $\mathrm{N}(\%)$ & $\mathrm{N}(\%)$ & $\mathrm{N}(\%)$ & $\mathrm{N}(\%)$ & $\mathrm{N}(\%)$ & $\mathrm{N}(\%)$ & $\mathrm{X}^{2}$ & $\begin{array}{l}\mathrm{P}- \\
\text { value }\end{array}$ \\
\hline Coat pattern & & & & & & & 45.68 & $<.0001$ \\
\hline Plain & $10(33.3)$ & $89(29.7)$ & $99(31.5)$ & $18(60)$ & $118(39.3)$ & $136(49.7$ & & \\
\hline Patchy & $19(63.3)$ & $180(60)$ & $199(61.7)$ & $10(33.3)$ & $107(35.7)$ & $117(34.5$ & & \\
\hline Spotty & $1(3.3)$ & $31(10.3)$ & $32(6.8)$ & $2(6.7)$ & $75(25)$ & $77(15.9)$ & & \\
\hline Coat type & & & & & & & 212.93 & $<.0001$ \\
\hline White & $3(10)$ & $24(8)$ & $27(9)$ & $15(50)$ & $21(7)$ & $36(28.5)$ & & \\
\hline Dark red & - & $6(2)$ & $6(1)$ & $1(3.3)$ & $34(11.3)$ & $35(7.3)$ & & \\
\hline Black & - & $12(4)$ & $12(2)$ & - & $13(4.3)$ & $13(2.2)$ & & \\
\hline Grey & $3(10)$ & $9(3)$ & $12(6.5)$ & - & $30(10)$ & $30(5)$ & & \\
\hline Light red & $1(3.3)$ & $8(2.7)$ & $9(3)$ & $2(6.7)$ & $30(10)$ & $32(8.4)$ & & \\
\hline Brown & $2(6.7)$ & $15(5)$ & $17(6)$ & $3(10)$ & $65(21.7)$ & $68(15.9)$ & & \\
\hline Brown and white & - & $7(2.3)$ & $7(1.2)$ & $6(20)$ & $17(5.7)$ & $23(12.9)$ & & \\
\hline $\begin{array}{l}\text { Brown dominate } \\
\text { with white }\end{array}$ & $4(13.3)$ & $12(4)$ & $16(8.7)$ & - & $17(5.7)$ & $17(2.9)$ & & \\
\hline $\begin{array}{l}\text { White dominate } \\
\text { with brown }\end{array}$ & $1(3.3)$ & $10(3.3)$ & $11(3.3)$ & - & $30(10)$ & $30(5)$ & & \\
\hline $\begin{array}{l}\text { White dominate } \\
\text { with black }\end{array}$ & $8(26.7)$ & $98(32.7)$ & $106(29.7)$ & - & $(16(5.3)$ & $16(2.7)$ & & \\
\hline $\begin{array}{l}\text { Black dominate } \\
\text { with white }\end{array}$ & $5(16.7)$ & $81(27)$ & $86(21.9)$ & $2(6.7)$ & $10(3.3)$ & $12(5)$ & & \\
\hline $\begin{array}{l}\text { Light red with } \\
\text { white spotty }\end{array}$ & $1(3.3)$ & $11(3.7)$ & $12(3.5)$ & - & $10(3.3)$ & $10(1.7)$ & & \\
\hline Dark grey & $2(6.7)$ & $7(2.3)$ & $9(4.5)$ & $1(3.3)$ & $7(2.3)$ & $8(2.8)$ & & \\
\hline Head profile & & & & & & & 15.01 & 0.0001 \\
\hline Straight & $11(36.7)$ & $114(38)$ & $125(37.4)$ & $8(26.7)$ & $71(23.7)$ & $79(23.9)$ & & \\
\hline Concave & $19(63.3)$ & $186(62)$ & $205(62.7)$ & $22(73.3)$ & $229(76)$ & $251(76.1)$ & & \\
\hline Back profile & & & & & & & 24.61 & $<.0001$ \\
\hline Straight & $10(33.3)$ & $186(62)$ & $196(47.7)$ & $23(76.7)$ & $230(76.7)$ & $253(76.7)$ & & \\
\hline $\begin{array}{l}\text { Slopes up towards } \\
\text { the rump }\end{array}$ & $15(50)$ & $83(27.7)$ & $97(38.9)$ & $5(16.7)$ & $45(15)$ & $50(15.9)$ & & \\
\hline $\begin{array}{l}\text { Slopes down from } \\
\text { the withers }\end{array}$ & $5(16.7)$ & $13(4.3)$ & $18(10.5)$ & $1(3.3)$ & $15(5)$ & $16(4.2)$ & & \\
\hline Curved & - & $18(6)$ & $18(3)$ & $1(3.3)$ & $10(3.3)$ & $11(3.3)$ & & \\
\hline
\end{tabular}




\begin{tabular}{|c|c|c|c|c|c|c|c|c|}
\hline Wattle & & & & & & & 1.31 & 0.2522 \\
\hline Presence & $9(30)$ & $30(10)$ & $39(11.8)$ & $3(10)$ & $27(9)$ & $30(9.1)$ & & \\
\hline Absent & $21(70)$ & $270(90)$ & $291(88.2)$ & $27(90)$ & 273(91) & $300(90.9)$ & & \\
\hline Beard & & & & & & & 0.03 & 0.8691 \\
\hline Present & $20(66.7)$ & $90(30)$ & $110(48.4)$ & $26(86.7)$ & $79(26.4)$ & $105(31.9)$ & & \\
\hline Absent & $10(33.3)$ & $210(70)$ & $220(51.7)$ & $4(13.3)$ & $220(73.6)$ & $224(68.1)$ & & \\
\hline Ruff & & & & & & & 0.37 & 0.3677 \\
\hline Present & $20(66.7)$ & $67(22.3)$ & $87(26.4)$ & $22(73.3)$ & $61(20.3)$ & $83(25.2)$ & & \\
\hline Absent & $10(33.3)$ & $233(77.7)$ & $243(73.6)$ & $8(26.7)$ & $239(79.7)$ & $247(74.8)$ & & \\
\hline Toggle & & & & & & & 38.35 & $<.0001$ \\
\hline Presence & $5(16.7)$ & $30(10)$ & $35(10.6)$ & $10(33.3)$ & $59(19.7)$ & $69(20.9)$ & & \\
\hline Absent & $25(83.3)$ & $270(90)$ & $395(89.4)$ & $20(66.7)$ & $241(80.3)$ & $261(79.1)$ & & \\
\hline Ear formation & & & & & & & 143.39 & $<.0001$ \\
\hline Forward & $8(26.7)$ & $28(9.3)$ & $36(18)$ & $10(33.3)$ & $151(50.3)$ & $161(41.8)$ & & \\
\hline Pendulous & - & - & - & - & $15(5)$ & $15(2.5)$ & & \\
\hline Semi-pendulous & $21(70)$ & $202(87.3)$ & $223(78.7)$ & $20(66.7)$ & $134(44.7)$ & $154(55.7)$ & & \\
\hline $\begin{array}{l}\text { Carried } \\
\text { horizontally }\end{array}$ & $1(3.3)$ & $10(3.3)$ & $11(3.3)$ & - & - & - & & \\
\hline Horn shape & & & & & & & 32.15 & $<.0001$ \\
\hline Straight & $21(70)$ & $224(79.2)$ & $245(78.8)$ & $22(73.3)$ & $185(61.9)$ & $207(62.9)$ & & \\
\hline Curved & $6(20)$ & $55(19.4)$ & $61(19.6)$ & $5(16.7)$ & $105(35.1)$ & $110(33.4)$ & & \\
\hline Spiral & $1(10)$ & $4(1.4)$ & $5(1.6)$ & $3(10)$ & $9(3)$ & $12(3.6)$ & & \\
\hline Horn formation & & & & & & & 6.50 & 0.0387 \\
\hline Upward & - & $30(10.6)$ & $30(9.6)$ & $3(10)$ & $51(17.1)$ & $54(16.4)$ & & \\
\hline Back ward & $24(85.7)$ & $239(84.5)$ & $263(84.6)$ & $26(86.7)$ & $233(77.9)$ & $259(78.7)$ & & \\
\hline $\begin{array}{l}\text { Back ward but the } \\
\text { tip bend to } \\
\text { forward }\end{array}$ & $4(14.3)$ & $14(4.9)$ & $18(5.8)$ & $1(3.3)$ & $15(5)$ & $16(4.9)$ & & \\
\hline Horn presence & & & & & & & 16.70 & $<.0001$ \\
\hline Present & $28(93.3)$ & $283(94.3)$ & $311(94.2)$ & $30(100)$ & 299(99.7) & $329(99.9)$ & & \\
\hline Absent & $2(6.7)$ & $17(5.7)$ & $19(5.8)$ & - & $9(0.3)$ & $1(0.1)$ & & \\
\hline
\end{tabular}

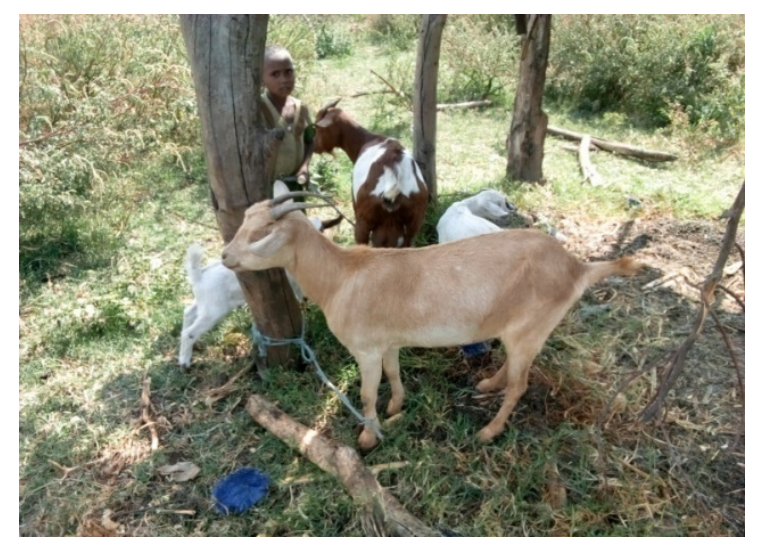

Figure 1.Female goat in Guraferda

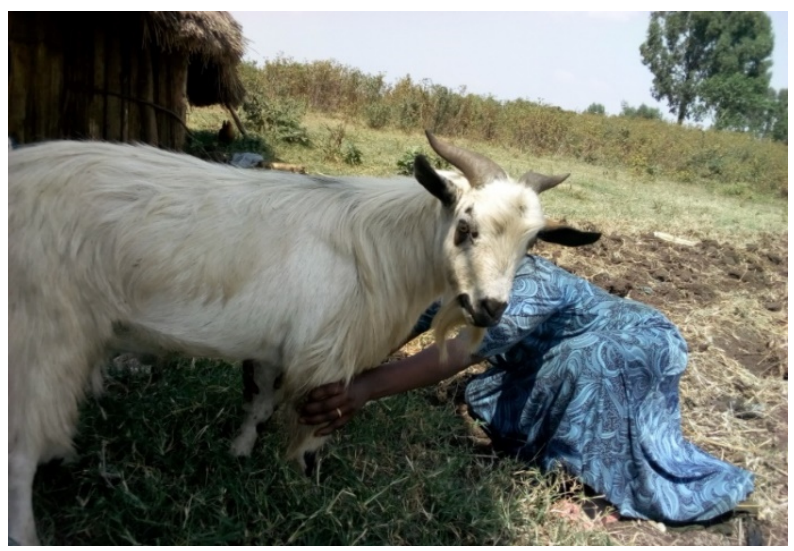

Figure2.Male goat in Guraferda 


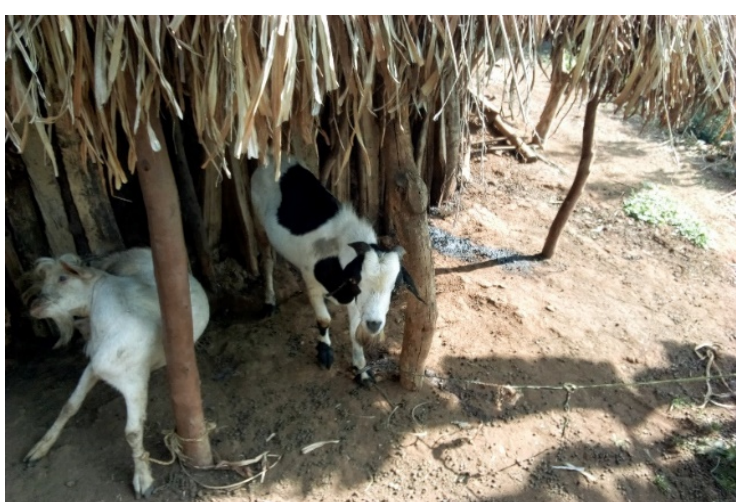

Figure 3.Male goats in Meanit Goldiya

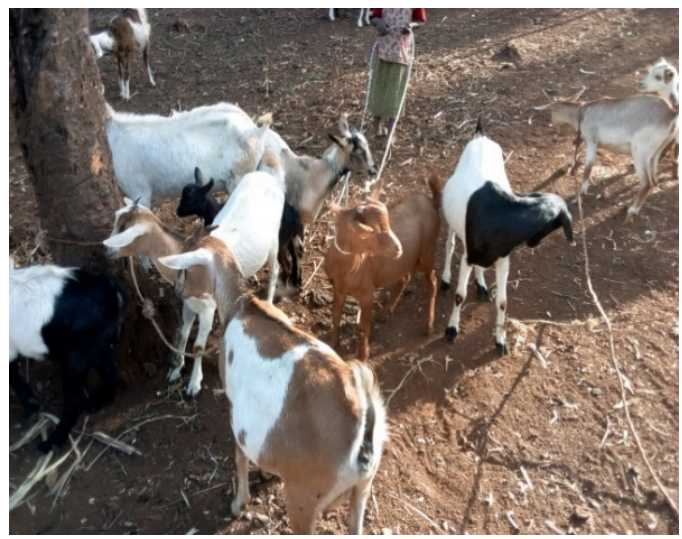

Figure 4.Female goats in Meanit Goldiya

Table2. means $(\mathrm{M})$ and standard error $( \pm \mathrm{SE})$ of body weight $(\mathrm{kg})$ and linear body weight measurement $(\mathrm{cm})$ for effects of district, sex, age group and sex and interaction of goats $(\mathrm{Male}=60, \mathrm{Fmale}=600)$

\begin{tabular}{|c|c|c|c|c|c|c|c|c|}
\hline Effect and level & BW & $\mathrm{BL}$ & CG & $\mathrm{WH}$ & $\mathrm{PW}$ & $\mathrm{RH}$ & $\mathrm{HOL}$ & $\mathrm{HL}$ \\
\hline & Mean \pm SE & Mean \pm SE & Mean \pm SE & Mean \pm SE & Mean \pm SE & Mean \pm SE & Mean \pm SE & Mean \pm SE \\
\hline Overall & $27.3 \pm 0.18$ & $60.1 \pm 0.16$ & $70.7 \pm 0.16$ & $60.2 \pm 0.11$ & $14.6 \pm 0.05$ & $62.3 \pm 0.08$ & $9.2 \pm 0.08$ & $11.06 \pm 0.05$ \\
\hline CV (\%) & 9.5 & 4.2 & 3.6 & 3.6 & 5.4 & 2.5 & 16.14 & 9.7 \\
\hline $\mathrm{R}^{2}$ & 0.68 & 0.62 & 0.61 & 0.47 & 0.68 & 0.51 & 0.40 & 0.39 \\
\hline District & ** & $* *$ & * & $* *$ & $* *$ & * & $* *$ & $* *$ \\
\hline Meanit Goldiya & $26.46 \pm 0.20^{\mathrm{a}}$ & $58.6 \pm 0.16^{\mathrm{a}}$ & $70.55 \pm 0.21^{\mathrm{a}}$ & $59.65 \pm 0.11^{\mathrm{a}}$ & $13.98 \pm 0.06^{\mathrm{a}}$ & $61.87 \pm 0.11^{\mathrm{a}}$ & $9.88 \pm 0.08^{\mathrm{a}}$ & $10.87 \pm 0.06^{\mathrm{a}}$ \\
\hline Guraferda & $28.19 \pm 0.28^{b}$ & $61.65 \pm 0.24^{\mathrm{b}}$ & $70.94 \pm 0.24^{\mathrm{b}}$ & $60.82 \pm 0.20^{\mathrm{b}}$ & $15.13 \pm 0.08^{\mathrm{b}}$ & $62.78 \pm 0.13^{\mathrm{b}}$ & $8.59 \pm 0.12^{\mathrm{b}}$ & $11.24 \pm 0.09^{\mathrm{b}}$ \\
\hline Sex & * & * & * & * & * & * & * & NS \\
\hline Male & $29.91 \pm 0.67^{\mathrm{a}}$ & $62.24 \pm 0.68^{\mathrm{a}}$ & $72.70 \pm 0.56^{\mathrm{a}}$ & $63.05 \pm 0.40^{\mathrm{a}}$ & $14.06 \pm 0.19^{\mathrm{a}}$ & $64.16 \pm 0.34^{\mathrm{a}}$ & $11.38 \pm 0.26^{\mathrm{a}}$ & $11.12 \pm 0.26$ \\
\hline Female & $27.07 \pm 0.18^{\mathrm{b}}$ & $59.91 \pm 0.16^{\mathrm{b}}$ & $70.55 \pm 0.17^{b}$ & $59.96 \pm 0.11^{\mathrm{b}}$ & $14.61 \pm 0.06^{\mathrm{b}}$ & $62.13 \pm 0.08^{\mathrm{b}}$ & $8.98 \pm 0.07^{\mathrm{b}}$ & $11.05 \pm 0.06$ \\
\hline Age group & $* *$ & $* *$ & $* *$ & $* *$ & $* *$ & $* *$ & $* *$ & $* *$ \\
\hline 1PPI & $20.49 \pm 0.14^{\mathrm{a}}$ & $55.15 \pm 0.26^{\mathrm{a}}$ & $64.20 \pm 0.24^{\mathrm{a}}$ & $56.37 \pm 0.17^{\mathrm{a}}$ & $12.59 \pm 0.11^{\mathrm{a}}$ & $59.48 \pm 0.29^{\mathrm{a}}$ & $7.96 \pm 0.22^{\mathrm{a}}$ & $9.67 \pm 0.19^{\mathrm{a}}$ \\
\hline 2PPI & $23.94 \pm 0.40^{\mathrm{b}}$ & $57.04 \pm 0.43^{b}$ & $68.27 \pm 0.42^{\mathrm{b}}$ & $59.06 \pm 0.24^{\mathrm{b}}$ & $13.61 \pm 0.09^{\mathrm{b}}$ & $61.10 \pm 0.23^{b}$ & $9.05 \pm 0.16^{\mathrm{b}}$ & $10.68 \pm 0.19^{\mathrm{b}}$ \\
\hline 3PPI & $26.70 \pm 0.23^{\mathrm{c}}$ & $59.81 \pm 0.25^{\mathrm{c}}$ & $70.25 \pm 0.19^{c}$ & $60.72 \pm 0.12^{\mathrm{c}}$ & $14.38 \pm 0.07^{\mathrm{c}}$ & $61.41 \pm 0.13^{\mathrm{c}}$ & $8.70 \pm 0.16^{\mathrm{ab}}$ & $10.91 \pm 0.10^{\mathrm{c}}$ \\
\hline 4PPI & $30.31 \pm 0.16^{\mathrm{d}}$ & $62.41 \pm 0.15^{\mathrm{d}}$ & $73.34 \pm 0.14^{\mathrm{d}}$ & $61.31 \pm 0.17^{\mathrm{d}}$ & $15.41 \pm 0.06^{\mathrm{d}}$ & $62.34 \pm 0.08^{\mathrm{d}}$ & $9.8 \pm 0.09^{\mathrm{c}}$ & $11.78 \pm 0.05^{\mathrm{d}}$ \\
\hline Age group by sex & $*$ & * & NS & $*$ & NS & * & * & NS \\
\hline $1 \mathrm{pp}$, female & $20.23 \pm 0.12^{\mathrm{a}}$ & $54.87 \pm 0.23^{\mathrm{a}}$ & $64.01 \pm 0.25$ & $56.00 \pm 0.08^{\mathrm{a}}$ & $12.61 \pm 0.12$ & $59.28 \pm 0.31^{\mathrm{a}}$ & $7.83 \pm 0.23^{\mathrm{a}}$ & $9.28 \pm 0.14$ \\
\hline 2PP, female & $23.60 \pm 0.44^{b}$ & $56.78 \pm 0.44^{b}$ & $67.98 \pm 0.47$ & $58.83 \pm 0.26^{\mathrm{b}}$ & $13.64 \pm 0.10$ & $60.96 \pm 0.26^{b}$ & $8.72 \pm 0.15^{\mathrm{b}}$ & $10.41 \pm 0.16$ \\
\hline 3PP, female & $26.15 \pm 0.18^{\mathrm{c}}$ & $59.35 \pm 0.22^{\mathrm{c}}$ & $69.81 \pm 0.16$ & $60.38 \pm 0.20^{\mathrm{c}}$ & $15.41 \pm 0.07$ & $62.16 \pm 0.12^{\mathrm{c}}$ & $8.28 \pm 0.13^{\mathrm{ab}}$ & $10.75 \pm 0.09$ \\
\hline 4PP, female & $30.04 \pm 0.16^{\mathrm{d}}$ & $62.21 \pm 0.15^{\mathrm{d}}$ & $73.13 \pm 0.14$ & $60.99 \pm 0.16^{\mathrm{c}}$ & $15.41 \pm 0.06$ & $63.11 \pm 0.06^{\mathrm{d}}$ & $9.63 \pm 0.06^{\mathrm{c}}$ & $11.78 \pm 0.06$ \\
\hline 1PP, male & $23.39 \pm 0.29^{\mathrm{ab}}$ & $57.15 \pm 1.18^{\mathrm{abc}}$ & $65.55 \pm 0.85$ & $59.00 \pm 0.87^{\mathrm{bc}}$ & $12.50 \pm 0.25$ & $60.95 \pm 0.63^{\mathrm{bc}}$ & $8.80 \pm 0.74^{\mathrm{abcd}}$ & $9.83 \pm 0.24$ \\
\hline 2PP, male & $26.38 \pm 0.81^{\mathrm{c}}$ & $58.88 \pm 1.46^{\mathrm{abc}}$ & $70.34 \pm 0.49$ & $60.67 \pm 0.27^{\mathrm{bc}}$ & $13.40 \pm 0.21$ & $62.08 \pm 0.28^{\mathrm{bcd}}$ & $11.13 \pm 0.52^{\mathrm{d}}$ & $10.75 \pm 0.21$ \\
\hline 3PP, male & $31.59 \pm 0.99^{\mathrm{d}}$ & $63.89 \pm 1.14^{\mathrm{d}}$ & $74.13 \pm 0.63$ & $63.67 \pm 0.34^{\mathrm{d}}$ & $15.38 \pm 0.13$ & $64.59 \pm 0.37^{\mathrm{e}}$ & $12.11 \pm 0.40^{\mathrm{d}}$ & $11.11 \pm 0.25$ \\
\hline 4PP, male & $34.03 \pm 0.55^{\mathrm{f}}$ & $65.19 \pm 0.78^{\mathrm{d}}$ & $76.20 \pm 0.22$ & $65.75 \pm 0.39^{\mathrm{d}}$ & $15.38 \pm 0.29$ & $66.45 \pm 0.34^{\mathrm{f}}$ & $12.16 \pm 0.24^{\mathrm{d}}$ & $11.91 \pm 0.18$ \\
\hline \multicolumn{9}{|l|}{ Table 1(continued) } \\
\hline \multirow[t]{2}{*}{ Effect and level } & EL & $\mathrm{CW}$ & \multirow{2}{*}{\multicolumn{2}{|c|}{$\begin{array}{l}\text { SC } \\
\text { Mean } \pm \text { SE }\end{array}$}} & \multirow{2}{*}{$\begin{array}{r}\text { Effect } \\
\text { level }\end{array}$} & EL & $\mathrm{CW}$ & $\mathrm{SC}$ \\
\hline & Mean \pm SE & Mean \pm SE & & & & Mean \pm SE & Mean \pm SE & Mean \pm SE \\
\hline Overall & $12.39 \pm 0.04$ & $14.4 \pm 0.05$ & \multicolumn{2}{|r|}{$23.8 \pm 0.18$} & $\begin{array}{l}\text { Age group by } \\
\operatorname{sex}\end{array}$ & NS & NS & \\
\hline $\mathrm{CV} \%$ & 5.4 & 8.6 & \multicolumn{2}{|r|}{2.3} & $1 \mathrm{pp}$, female & $11.79 \pm 0.11$ & $12.68 \pm 0.16$ & \\
\hline $\mathrm{R}^{2}$ & 0.47 & 0.29 & \multicolumn{2}{|r|}{0.87} & $2 \mathrm{PP}$, female & $11.98 \pm 0.08$ & $13.70 \pm 0.12$ & \\
\hline District & $* *$ & $* *$ & \multicolumn{2}{|r|}{ NS } & 3PP, female & $12.52 \pm 0.07$ & $14.32 \pm 0.49$ & \\
\hline Meanit Goldiya & $11.84 \pm 0.05^{\mathrm{a}}$ & $14.15 \pm 0.06^{\mathrm{a}}$ & \multirow{2}{*}{\multicolumn{2}{|c|}{$23.86 \pm 0.24$}} & 4PP, female & $12.50 \pm 0.05$ & $14.88 \pm 0.07$ & \\
\hline Guraferda & $12.93 \pm 0.03^{\mathrm{b}}$ & $14.59 \pm 0.09^{\mathrm{b}}$ & & & $1 \mathrm{PP}$, male & $11.99 \pm 0.29$ & $13.30 \pm 0.54$ & $21.30 \pm 0.19$ \\
\hline Sex & NS & * & & & 2PP, male & $12.44 \pm 0.12$ & $14.19 \pm 0.49$ & $23.27 \pm 0.23$ \\
\hline Male & $12.35 \pm 0.04$ & $14.85 \pm 0.25^{\mathrm{a}}$ & \multicolumn{2}{|r|}{$23.82 \pm 0.19$} & 3PP, male & $12.97 \pm 0.09$ & $15.23 \pm 0.10$ & $23.97 \pm 0.07$ \\
\hline Female & $12.77 \pm 0.08$ & $14.33 \pm 0.06^{\mathrm{b}}$ & \multicolumn{2}{|r|}{ - } & 4PP, male & $13.15 \pm 0.06$ & $15.65 \pm 0.36$ & $25.20 \pm 0.11$ \\
\hline Age group & $* *$ & $* *$ & \multicolumn{2}{|r|}{$* *$} & & & & \\
\hline 1PPI & $11.81 \pm 0.10^{\mathrm{a}}$ & $12.76 \pm 0.15^{\mathrm{a}}$ & & & & & \\
\hline 2PPI & $12.04 \pm 0.07^{\mathrm{a}}$ & $13.76 \pm 0.12^{\mathrm{b}}$ & \multirow{2}{*}{\multicolumn{2}{|c|}{$\begin{array}{l}23.22 \pm 0.23^{\mathrm{b}} \\
23.96 \pm 0.07^{\mathrm{c}}\end{array}$}} & & & & \\
\hline 3PPI & $12.56 \pm 0.07^{\mathrm{b}}$ & $14.41 \pm 0.11^{\mathrm{c}}$ & & & & & & \\
\hline 4PPI & $12.55 \pm 0.05^{\mathrm{c}}$ & $14.94 \pm 0.07^{\mathrm{d}}$ & \multicolumn{2}{|r|}{$\begin{array}{l}23.96 \pm 0.07^{\mathrm{c}} \\
25.20 \pm 0.11^{\mathrm{d}}\end{array}$} & & & & \\
\hline
\end{tabular}

Means with different superscripts within the same column and class are statistically different (at least $P<0.05$ ).Ns $=$ Non-significant $(\mathrm{P}>0.05) ; *$ significant at $0.05 ; * *$ significant at $0.01 ; \mathrm{BW}=\mathrm{Body}$ weight, $\mathrm{CG}=\mathrm{Heart}$ girth, $\mathrm{HW}=$ Height at whither; $\mathrm{RH}=$ Rump height; $\mathrm{BL}=$ Body length; $\mathrm{EL}=$ Ear length; $\mathrm{PW}=$ Pelvic width; $\mathrm{HOL}=$ horn length; $\mathrm{CW}=$ chest width; $\mathrm{HL}=$ Head length; $\mathrm{SC}=$ Scrota circumference; $1 \mathrm{PPI}=1$ Pair of Permanent Incisors; $2 \mathrm{PPI}=$ 2Pairs of Permanent Incisors; 4PPI $=4$ pair of permanent incisors.

The present result is in agreement with the earlier report, almost all goats possessed horn in Nure zone Gambella (Tsigabu, 201-5), central high land (Netsanet, 2014) and Bench Maji area (Fantahun et al., 2013). The result obtained for straight horn shape and back ward direction is in consistent with the report of Fantahun et al. (2013) in Bench Maji zone, south western Ethiopia. The variation in this study might be resulted due to breed and geographical differences.

Determine animal live weight, linear body measurement and their relationship are very important for determine genetic potential, breed standards and improving breeding programs for higher meat production (Younas et al.,2013). The difference in live body weight between male and female across different age class indicate that these parameters are sex and age dependent. The report of Grum (2010) in Woyto-Guji goat, Halima et al. (2012) in Amhara region, Mahilet (2012) in Hararghe highland and Fantahun et al. (2013) in Bench Maji zone who 
reported that location, sex and age differences were apparent. The value obtained for body weight $(27.3 \pm 0.18 \mathrm{~kg})$ in this study was in com-parable with the result of Solomon et al. (2009) and Alubel (2015) who reported that the average body weight was $27.8 \mathrm{~kg}$ and $27.52 \mathrm{~kg}$ for short eared Somali and Aberg-elle goats, respectively. Likely, the present result is closer to the work of Solomon et al. (2009) stated that the body weight of Abergelle and Woyto-Guji goats were $28.4 \mathrm{~kg}$ and $28.8 \mathrm{~kg}$, respectively. However, this value higher than the report of Mahilet (2012) and Solomon et al. (2009) revealed that the average body weight of Haraghe high land and Afar goats were $24.48 \mathrm{~kg}$ and $23.7 \mathrm{~kg}$, respectively. Similarly, Solomon et al. (2009) who showed that the body weight of Arsi Bale and long eared Somali goats were $30.4 \mathrm{~kg}$ and $31.8 \mathrm{~kg}$, respectively which were higher than the current findings. Seifemichael (2013) reported that the body length of Afar goats was $60.69 \pm 0.02 \mathrm{~cm}$ which was in comparable with the present finding. Likewise; Alubel (2015) revealed that the body length of Abergelle goats was $61.44 \pm 0.30 \mathrm{~cm}$ which was in closer with the present result. On the other hand, the current value was higher than the earlier report of Hulunium (2014) reported that the body length of short eared Somali goats was $57.85 \pm 0.41 \mathrm{~cm}$. According to Tsigabu (2015) reported that the average body length of Nure goats was $56.93 \pm 3.64 \mathrm{~cm}$ which was lower than the current result. The mean value obtained for chest girth was compare-able to the earlier report of Mahilet (2012), Alubel (2015) disclosed that the average chest girth of Haraghe high land and Aberg-elle goats were $70.4 \mathrm{~cm}$ and69.32 cm, respectively. In the same way, this value was in comparable with the work of Solomon et al. (2009) reported that the chest girth of sort eared Somali, long eared Somali and Abergelle goats were $70.4 \mathrm{~cm}, 70.4 \mathrm{~cm}$ and $71.2 \mathrm{~cm}$, respectively. However, this value was lower than the previous study, Solomon et al. (2009) reported that the average chest girth of Woyto-Guji and Arsi-Bale and keffa goats were $72.5 \mathrm{~cm}$ and $74.9 \mathrm{~cm}$ and $72.2 \mathrm{~cm}$, respectively. However, the current value was higher than the chest girth of Afar goat which was $67.4 \mathrm{~cm}$ (Solomon et al., 2009). Mahilet (2012) reported that the wither height of goats reared in Haraghe high land was $(61.91 \mathrm{~cm})$ and Solomon et al. (2009) for Afar $(60.9 \mathrm{~cm})$, short eared Somali $(61.8 \mathrm{~cm})$ and keffa $(66.7 \mathrm{~cm})$ goat population which was in comparable with the current result. However, the current linear measure-ment value was lower than the report of Solomon et al. (2009) for Abergelle $(65 \mathrm{~cm})$, Arsi-Bale $(66.1 \mathrm{~cm})$ and Woyto-Guji $(66.4 \mathrm{~cm})$ and Alubel (2015) for Abergelle $(65.31 \mathrm{~cm})$ goat population. According to the report of Solomon et al. (2009) reported that goats reared in Afar, Abergelle, Woyto-Guji part of Ethiopia had ear length measu-res $12.3 \mathrm{~cm}, 12.7 \mathrm{~cm}$ and $12.5 \mathrm{~cm}$, respectively which was in agreement with this study result. Similarly, by Solomon et al. (2009) revealed that the horn length of long ear Somali and woyto-Guji goats were $9 \mathrm{~cm}$ and $10.8 \mathrm{~cm}$, respectively which was in accordance with the current findings. However, the current findings were lower than the previous report of Solomon et al. (2009) for keffa goats $13 \mathrm{~cm}$ and Arsi-Bale $14 \mathrm{~cm}$ of ear length and horn length $11.6 \mathrm{~cm}$ and 12.5 for keffa and Arsi-Bale goats, respectively. This value is in comparable with the findings of Hulunim (2014) for Bati, Borena and Short eared Somali goats were $14.36 \pm 0.09 \mathrm{~cm}, 14.17 \pm 0.07 \mathrm{~cm}$ and $13.73 \mathrm{~cm}$, respectively. However, the present result was higher than the work of Belete (2013) and Tadesse (2012) $9.52 \pm 0.04 \mathrm{~cm}$ and $13.21 \pm-1.46 \mathrm{~cm}$ for Arsi-Bale and Woyto-Guji goats, respectively. The previo-us report of Belay and Meseretu (2017) reported that the scrotum circumference of Gamo Gofa goat was $23.53 \pm 0.18 \mathrm{~cm}$ which was comparable for the value obtained for SC. Nevertheless, this result was higher than the scrotum circumference of West Gojjam goats $(21.26 \pm 0.18 \mathrm{~cm})$ (Bekalu et al., 2016) and Woyto-Guji goat (16.56 $\pm 2.77 \mathrm{~cm}$ ) (Lorato et al., 2015).Rump height $(62.3 \pm-0.08 \mathrm{~cm})$ in the current study is in closer with the report of Mahilet (2012) for Hararghe high land goat $(63.32 \pm 0.17 \mathrm{~cm})$ and $61.1 \pm-0.03 \mathrm{~cm}$ for Afar goats (Seifemichael, 2013). The value obtained from the current study for head length $(11.06 \pm 0.05 \mathrm{~cm})$ was comparable to those reported by Netsanet (2014) for Woyto-Guji goats $(10 \pm-0.2 \mathrm{~cm})$ population but was lower than those report-ed by Hulunuim(2014) for Bati goats $(13.87 \pm 0.24)$ and $12.8 \pm 0.2$ for central high land goats (Netsanet,2014). Alubel (2015) reported that goats reared in Abergelle part of Ethiopia had chest width measure $14.24 \pm 0.11 \mathrm{~cm}$ which is in closer the result obtained from the current finding of chest width $(14.4 \pm 0.05 \mathrm{~cm})$. But which was higher than the chest width of Woyto-Guji $(12.1 \pm-0.1 \mathrm{~cm})$ and $13.4 \pm 0.1$ $\mathrm{cm}$ for central high land goats reported by (Netsanet, 2014-). Similarly, Hulunuim (2014) stated that the chest width of Bati, Borena and short eared Somali goats were $17.10 \pm 0.16 \mathrm{~cm}, 16.37 \pm-0.12 \mathrm{~cm}$ and $15.35 \pm 0.14 \mathrm{~cm}$, respectively which was higher than the current study finding. The variation of the body measure-ment amongst Ethiopian goats could be due to breed, location difference and feed availability generally husbandry practices. The value obtained for female and male goat in the study area was in comparable with the previous work of Fantahun et al. (2013) report that the body weight of males and females goats in Bench Maji area was $31.02 \pm 0.5$ and $26.36 \pm 0.21 \mathrm{~kg}$, respectively. However, Belay and Meseretu (2017) who reported $26.17 \pm 0.33 \mathrm{~kg}$ for male which is lower than the result found for male goats and $28.61 \pm 0.23 \mathrm{~kg}$ for female goats of Gamo Gofa zone which is higher than the value obtained for female goats. In the current study result male goats have higher body weight than female goats which was in agreement with the report of Alemayehu et al. (2012) and Gebreyesus et al. (2012) disclosed that where males have higher body weight than female counterpart for Abergelle and Short Eared Somali goat. However, the result is not in consistent with the work of (Alade et al., 2008; Sowande et al.,2009; Semakula et al.,2010 and Okbeku et al. ,2011) stated that females have higher body weight and other body measurement than male counterpart. The probable reason might be related with the usual differences between sex hormonal effects which lead to differential growth rates (Semakula et al, 2010). It was revealed that does have slower rate 
of growth and reach maturity at smaller size due to the effect of estrogen in restricting the growth of the long bones of the body (Sowande and Sobola, 2007). The liner body measurement increased as animal advances with age (1PPI to 4PPI), which was similar with the report of Mohammed et al. (2017) reported that, body weight and all linear body measurements were significantly influenced by age group and body weight and other linear body measurements was increase with the age of animals; Kenfo et al. (2017) reported that body weight increase with an increase in age of the animals. The result obtained for 1PPI body weight $(20.49 \pm 0.14 \mathrm{~kg})$ was in closer with the study of Lorat et al. (2015) who stated that the body weight of 1PPI age group of Woyto- Guji goat was $20.38 \pm 0.36$ $\mathrm{kg}$. Similarly, Mahilet (2012) showed that the body weight of 1PPI was $20.59 \pm 0.38 \mathrm{~kg}$ in Hararghe high land goats which was in compare-able with the current finding. Howe-ver, Solomon (2014) reported that the body weight of 1 PPI was $21.9 \mathrm{~kg}$ in Western lowland and Abergelle goats which is higher than the result of this study finding. The value obtained for 2PPI $(23.94 \pm 0.40 \mathrm{~kg})$ in this study was in comparable with the finding of Mahilet (20-12) disclose that the body weight of 2 PPI was $24.6 \pm 0.34 \mathrm{~kg}$ in Hararghe high land goats and this value slightly higher than the report of Solomon (2014) in Western lowland and Abergelle goats (23.07kg). The study of Solomon (2014 ) indicated that the body weight of 3PPI was $25.5 \mathrm{~kg}$ in Western lowland and Abergelle goats which was in comparable with this result $(26.7 \pm 0.23 \mathrm{~kg})$. Similarily,this result value was similar with the finding of Mahilet(2012) revealed that the body weight of 3PPI was $27.39 \pm 0.34 \mathrm{~kg}$ in Hararghe high land goats. The weight of goats at 4PPI was $30.31 \pm 0.16 \mathrm{~kg}$, which is similar to the report of Mahilet (2012) reported that the body weight at 4PPI of Hararghe highland goats was $29.67 \pm 0.72 \mathrm{~kg}$. This value also comparable with the work of Solomon (2014) showed that the body weight of 4PPI was $29.13 \mathrm{~kg}$ in Western lowland and Abergelle goats, which is greater than $23.07 \pm 0.2 \mathrm{~kg}$ reported for short Somali goat types (Grum, 2010).

The higher body weight of goats in Gurafer-da than Meanit Goldiya area might be might be due to management difference and feed shortage problem in Meanit Goldiya district than Guraferda district. The value obtained from these districts was higher than the earlier report of Dereje et al. (2013) who reported $23.9 \pm 4.66 \mathrm{~kg}$ for Hararghea highland goat, Gelana and Belete (2016) who reported $25 \pm 3.6 \mathrm{~kg}$ for Bale goat's and Biruh (2013) $22 \pm 0.2 \mathrm{~kg}$ for Woyto-Guji goat. In all age group males were heavier than females which is similar with short eared Somali goats, male goats were greater than their female counter parts in all age group and all measurement (Grum, 2010). similarly, Mahilet (2012) reported that male goat's performed greater than female counter parts in all age group and measurement in Hararghe highland goat which was in agreement with the present findings.

\section{Conclusion}

The findings of this study revealed that plain coat color pattern (in Guraferda), patchy (in Meanit Goldiya), concave head profile, straight back profile, semi-pendulous ear, straight horn shape with back ward direction were the predominant morphological traits of indigenous goats in the study districts. The most prevalent coat color type in Gurafreda area was white for male and brown for female, while in Meanit Goldiya white dominant with black coat color type occurring at comparable proportions. The population of indigenous goats studied showed heterogeneity in most morphological traits considered. The medium body weight was obtain-ed from this the study areas. Hence, information on morphological and morph metric is important for initiating the new breeding, management improvement and conservation program. It is thus recommended that the improvement strategies have to consider the trait that favors direct economic importance received from such goat population at traditional extensive environment and in-depth molecular evaluation is needed for prop-er utilization to further explore the potential of this genetic material through improving genetic and husbandry management system of goat.

\section{Reference}

Alade N, Raji, A and Atiku M (2008). Determination of appropriate model for the estimation of body weight in goats.Department of Animal Science, University of Maiduguri, Maiduguri, Borno State, Nigeria, ARPN Journal of Agricultural and Biological Science.3 (4).

Alubel, A (2015). On-farm phenotypic characterizations and performance eva-luation of Abergelle and Central highl-and goat breeds as an input for design-ning community-based breeding prog-ram's Thesis.Haramaya University, Ha-ramaya, Ethiopia.

Assen, E. \& Aklilu, H (2012). Sheep and goat production and utilization in differ-ent agro ecological zones in Tigray, Ethiopia. Livestock Research for Rural Development, 24

Bekalu M, Kefelegn K, Yoseph M (2016). Phenotypic Characterization of Indigen-ous Goat Types in West Gojjam Zone, Amhara National Regional State, Ethio-pia. Journal of Biology, Agriculture and Healthcare Vol.6 (6).

Belay Z and Meseretu M (2017). On Farm Phenotypic Characterization of Indige-nous Goat Populations in Gamo Gofa Zone South Western Ethiopia, Journal of Advanced Studies in Agricultural, Biological and Environmental Sciences (Jabe), Vol.4.-Issue.3.2017 (July-Sept).

Belete A (2013). On farm phenotypic characterization of indigenous goat types and their production system in Bale Zone of Oromia Region, Ethiopia. MSc thesis presented at Haramaya University.

Biruh T (2013). Phenotypic and Production System Characterization of Woyto Guji Goats in Low Land Areas of 
South Omo Zone. MSc thesis, Submitted to the School of Graduate Studies of Haramaya University, Ethiopia. CSA (2018). Agricultural Sample Survey of 2017/18 (2010 E.C). Volume II. Report on Livestock and Livestock Characteristics (Private Peasant Holdi-ngs), Central Statistical Agency, Addis Ababa, Ethiopia.

Dereje T, Berhanu B and Aynalem H (2013). Morphological Characterization of Indigenous Harar-ghe Highland Goat Breed in Their Native Environment, West Hararghe, Ethiopia. American-Eurasian J. of Sci. Res. 8 (2):72-79.

EBI (2016). Farm Animal Diversity of Ethiopia: Breeds and Ecotypes Catalo-gue. Ethiopian Biodiversity Institute (EBI), Addis Ababa, Ethiopia.

FAO (2010). Breeding strategies for sustainable management of animal genetic resources. Animal Production and Health Guide lines. Rome, Italy. http://www.fao.org/docrep/012/i1103e/i1103e.pdf

FAO (2012). Phenotypic characterization of animal genetic resources. FAO Animal Production and Health Guidelines No.11. Rome, Italy.

FAO (2011). Draft Guidelines on Phenot-ypic Characterization of Animal Genet-ic Resources. Rome, 18 - 22 July.

Gebreyesus, G., Haile, A \& Dessie, T (2012). Body weight prediction equati-onns from different linear measurements in the Short-Eared Somali goat population of Eastern Ethiopia. Resear-ch Journal of Animal Sciences, 6(4-6): 90-93.

Gelana J \& Belete A (2016). Characte-rization of Indigenous Goats Type using Morphological Characters and Body Measurements in Sinana District, Bale Zone, South East Ethiopia. GJSFR-D Agriculture and Veterinary Volume 16 Issue 7.

Girma D, Mieso G, Feyisa H and Misgana D, 2013.Assessment of farmers' manag-ement practices and factors affecting goats' production system in Adami Tulu Jido kombolcha district of East Shawa Zone, Ethiopia. Agric. Biol. J. N. Am., 2013, 4(5): 520-526

Grum G (2010). Community-Based Parti-cipatory Characterization of the short Eared Somali Goat Population Around Dire Dawa. An MSc thesis submitted to School of Animal and Range Science, School of Graduate Studies Haramaya University. 129pp.

Halima H, Samer L, Barbara R, Michael B and Markos T (2012). Phenotypic chara-cterizeation of Ethiopian indigenous goat populations. Afr. J. of Biotech.11 (73), 13838-1384

Halima, H., Michael, BM., Rischkowsky, B. and Tibbo, M (2012a). Phenotypic characterization of Ethiopian indigen-ous goat populations. African Journal of Biotechnology 11(73):13838-13846.

Hulunim G (2014). On-Farm Phenotypic Characterization and Performance Ev-aluation of Bati, Borena and ShortEared Somali Goat Populations of Ethiopia. MSc thesis, Submitted to the School of Graduate Studies of Haram-aya University, Ethiopia.

Kenfo, H., Mekasha, Y., Tadesse, Y (2017): On farm phenotypic character-rization of indigenous of sheep type in Bensa district, southern Ethiopia. Journal of biology, Agriculture and health-care.Vol.7, No.19, 2017.

Legesse, G., Abebe, G., Siegmud-Schultze, M. and Valle-Zárate, A (2008). Small ruminant production in two mixed-farming system of southern Ethiopia: Status and prospects for improvement. Experimental Agriculture, 44:399-412.

LMP (2015). Institutions and policies to implement the Ethiopia livestock master planEthiopiaLMPBrief.https://cgspace.cgiar.org/bitstream/handle/10568/68038/LMP_5.pdf?sequence=1.

Lorato, Y., Ahmed, K.M. and Belay, B. (2015). Participatory Characterization of the Woyto-Guji Goat and Its Production Environment around Northern Omo, Ethiopia. The Journal of Agriculture and Natural Resources Sciences, 2(2), 455-465.

Mahilet D (2012). Characterization of Hararghe Highland Goat and their Production System in Eastern Hararghe. MSc thesis, Submitted to the School of Graduate Studies of Haramaya Univer-sity, Ethiopia.

Manzi M, Rutagwenda T, Kanuya N, Chatikobo P (20110. Phenotypic characterization of goats raised under traditional husbandry systems in Buge-sera and Nyagatare districts of Rwanda. J. Anim. Vet. Adv 10(24):3297-3302.

Mohammed, N., Alemayehu, K., Getachew, T (2017). On-farm phenoty-pic characterization of indigenous sheep population and its cross with awassi in selected Districts of South Wollo, Amhara, Ethiopia. Trop. Drylands 1, 1-11. Doi:10.13057/tropdrylands/t010101

Netsanet, Z (2014). On-farm phenotypic characterizations and performance eval-uation of Central highland goat and Woyto-Guji goat types for designing community-based breeding strategies. MSc Thesis.Haramaya University, Har-amaya, Ethiopia.

Okbeku, M., Yakubu, A., Olusolapeters, S., Ozoje, M.O., Ikeobi, C.O., Adebambo, O.A. andImumorin, I.G (2011). Applic-ation of multivariate principal component analysis to morp-hological characterization of indigenous goats in southern Nigeria. Received July 22, 2011; accepted September 15.

Saico S, andAbul S (2007). Socio-economic constraints on goat farming in the lowland of Swaziland. J. Sustain. Dev. Afr. 9:37-49. 
SAS (Statistical Analysis System) institute inc. 2009. SAS procedure guide, Version 9.2. Cory, NC, USA.

Seifemichael M (2013). Phenotypic characterization of indigenous afar goat breed and husbandry practices of pastoralists in afar region. M.Sc.Thesis, Haramaya University, Haramaya, Ethio-pia.

Semakula, J., Mutetikka, D., Kugonza, R. D. and Mpairewe, D (2010). Variability in BodyMorphometric Measurements and Their Application in Predicting Live Body Weight of Mubende and Small East African Goat Breeds in Uganda. Middle-East Journal of Scientific Research 5 (2): pp98-105

Solomon G (2009). Goat breeds of Ethiopia. A guide for identification and utilization. Ethiopia Sheep and Goat Productivity Improvement Program (ESGPIP).

Solomon G, Azage T, Berhanu G and Dirk H (2010). Sheep and goat production and marketing systems in Ethiopia: Characteristics and strategies for impr-ovement. IPMS (Improving Produ-ctivity and Market Success) of Ethiopian Farmers Project Working Paper 23. ILRI (International Livestock Research Institute), Nairobi, Kenya. pp58.

Solomon AG (2014). Design of commu-nity based breeding programs for two indigenous goat breeds of Ethiopia. Doctoral Thesis: University of natural resources and applied life sciences, department of sustainable agricultural systems division of livestock sciences. Vienna, Austria.

Sowande O., Oyewale, B, Iyasere, O (2009). Age and sex dependent regression models for predicting the live weight of West African dwarf goat from body measurements. Tropical animal health production 42: pp969975.

Tadesse, A., Gebremariam, T \&Gangwar, S. K (2012). Application of linear body measurements for predicting body weight of Abergelle goat breed in Tigray region, Northern-Ethiopia. Global Journal of Bio Science and Biotechnology, 1(2): 314-319

Tegegn F, Kefyalew A and Solomon A (2013). Identif-ication and phenotypic characterization of goat ecotypes in the Bench Maji zone, southwestern Ethiopia Animal Genetic Resources, 2013, 53, 19-26. C) Food and Agriculture Organization of the United Nations

Tesfaye G (2008). Characterization of Menz and Afar indigenous sheep breeds of smallholders and pastoralists for designing community-based breeding strategies in Ethiopia. An MSc Thesis Presented to the School of Graduate Studies of Hramaya University, Hramaya. Pp155.

Tesfaye K (2010). Assessment of on-farm breeding practices and estimation of genetic and phenotypic parameters for reproductive and survival traits in indigenous Arsi-Bale goats. MSc thesis, Submitted to the School of Graduate Studies of Haramaya University, Ethiopia.

Tsegaye T (2009). Characterization of goat production systems and on- farm evaluation of the growth performance of Grazing goats supplemented with different protein Sources in metema woreda, Amhara region, Ethiopia. MSc. Thesis, School of Graduate Studies of Haramaya University.

Thiruvenkadan, a (2005). Determination of best-fitted regression model for estimation of body weight in Kanni Adu Kids under farmer's management system. Livestock research for RuralDevelopment.17: 1- 11.

Tsigabu G, Getahun G, Kefelegn K and Yosef M (2015). Phenotypic character-rization of goat type in Nuer Zone of Gambella People Regional State, South Western Ethiopia, Global Journal of Animal Breeding and Genetics Vol. 3 (5), pp. 164-180, August, 2015

Wilson, R. T. and J. W. Durkin (1984). Age at permanent incisor eruption in indigenous goats and sheep in semiarid Africa. Livestock Prod. Sci. 11(4):451-455.

Yakubu,A., Ladokun,A.O \&Adua,M.M (2011).Bioprediction of Body Weight from Zoometrical Traits of Nondescript Goats using Linear and Non-Linear Models in North Central Nigeria. Livestock Research for Rural Development, Volume 23, Article \#130. (Retrieved September 4, 2013, athttp://www.lrrd.org/lrrd23/6/yaku23130.htm

Younas U., Abdullah M, Bhatti. J. A., Pasha T. N, Ahmad N., Nasir M and Hussain A (2013). Inter-relationship of body weight with linear body measurem-ents in hissardale sheep at different stages of life the journal of animal \& plant sciences, 23(1): 2013, page: 40-44 ISSN: 1018-7081.http://www.thejaps.org.October 10, 2015. 